\title{
Safety of endoscopic retrograde cholangiopancreatography practice in a district hospital
}

\author{
Authors: Shuann Shwana ${ }^{\mathrm{A}}$ and Dlovan Taha ${ }^{\mathrm{B}}$
}

\section{Aims}

An audit was undertaken to provide accurate success and complication rates to patients undergoing endoscopic retrograde cholangiopancreatography (ERCP).

\section{Methods}

Consecutive procedures from a single ERCP endoscopist's practice were included. Data on indications, results and early complications were collected prospectively.

\section{Results}

101 procedures from a 6 -month period were studied. In 73 (73\%), the ampulla was intact (no earlier sphincterotomy). The majority of procedures (65\%) were undertaken for gallstone disease, $22 \%$ for suspected cancer, $3 \%$ for stent occlusion and $10 \%$ for other causes. In three cases cholangiography showed no pathology, but in each there had been a concern of choledocholithiasis. Magnetic resonance cholangiopancreatography (MRCP) was carried out on 48 cases. In $71 \%$ of the cases, the ERCP was primarily therapeutic.

In $35 \%$ of the cases, biliary cannulation failed. The procedure was abandoned in 24 cases (because of the pathology encountered, unable to cannulate the papilla, patient discomfort, unable to cannulate the common bile duct or because of full stomach).

There were no deaths during the immediate postoperative period. Complications occurred after 19 (19\%) of procedures. The commonest complication was acute pancreatitis. It occurred in $10(9.9 \%)$ patients, in nine of whom it was mild and quickly self-limiting. In the other patient, respiratory failure responded to conservative measures. No surgical or radiological intervention was required. There were no other complications such as bleeding, perforation or infection.

\section{Conclusions}

ERCP can be practised successfully in a district general hospital setting, but complication rates are not insignificant.

Authors: ${ }^{\text {APrince }}$ Charles Hospital, Merthyr Tydfil, UK; ${ }^{B}$ University Hospital of Wales, Cardiff, UK

\section{Conflict of interest statement}

None declared. 